Article

\title{
Improved Separation and Collection of Charge Carriers in Micro-Pyramidal-Structured Silicon/PEDOT:PSS Hybrid Solar Cells
}

\author{
Yuuki Sugano ${ }^{1}$, Keisuke Sato ${ }^{1, *}$, Naoki Fukata ${ }^{2}$ and Kenji Hirakuri ${ }^{1}$ \\ 1 Department of Electrical and Electronic Engineering, Tokyo Denki University, 5 Senju-Asahi-cho, Adachi-ku, \\ Tokyo 120-8551, Japan; 15kmj21@ms.dendai.ac.jp (Y.S.); hirakuri@mail.dendai.ac.jp (K.H.) \\ 2 International Center for Materials Nanoarchitectonics, National Institute for Materials Science, 1-1 Namiki, \\ Tsukuba, Ibaraki 305-0044, Japan; fukata.naoki@nims.go.jp \\ * Correspondence: satok@mail.dendai.ac.jp; Tel.: +81-3-5284-5406
}

Academic Editor: Senthilarasu Sundaram

Received: 5 February 2017; Accepted: 21 March 2017; Published: 23 March 2017

\begin{abstract}
Silicon (Si)/organic polymer hybrid solar cells have great potential for becoming cost-effective and efficient energy-harvesting devices. We report herein on the effects of polymer coverage and the rear electrode on the device performance of Si / poly(3,4-ethylenedioxythiophene): poly(styrenesulfonate) (PEDOT:PSS) hybrid solar cells with micro-pyramidal structures. These hybrid solar cells provided adequate generation of charge carriers owing to the suppression of reflectance to below $13 \%$. Additionally, the separation of the photogenerated charge carriers at the micro-pyramidal-structured Si/PEDOT:PSS interface regions and their collection at the electrodes were dramatically improved by tuning the adhesion areas of the PEDOT:PSS layer and the rear electrode materials, thereby attaining a power conversion efficiency of $8.25 \%$. These findings suggest that it is important to control the PEDOT:PSS coverage and to optimize the rear electrode materials in order to achieve highly efficient separation of the charge carriers and their effective collection in micro-textured hybrid solar cells.
\end{abstract}

Keywords: inorganic/organic hybrid solar cells; pyramidal-structured silicon; conjugated conducting polymer; photovoltaic performance; light-harvesting efficiency

\section{Introduction}

Photovoltaic cells have attracted attention as a useful renewable energy technology over the years because they can resolve some concerns such as the exhaustion of fossil fuels, global warming by the emission of greenhouse gases, and so on [1]. To develop such cells and enhance their commercial usage, the selection of materials is of crucial importance. Mono- and/or multi-crystalline silicon ( $\mathrm{Si}$ ) materials are key materials for high-performance solar cells [2]. The market share of Si-based solar cells is currently more than $80 \%$ because of their excellent advantages, e.g., high power conversion efficiency (PCE) [3] and long-term stability [4]. However, the cost of solar cells is approximately three times higher than that of fossil fuels [5]. This high cost has relevance to the expense of cell fabrication. Actually, cell fabrication accounts for as much as $30 \%$ of the total manufacturing cost owing to the need to use energy-intensive processes, such as ultrahigh vacuum physical vapor deposition [6], impurity diffusion using high-temperature furnaces [6], etc.

Organic polymeric materials are potentially effective tools for resolving many issues regarding Si-based solar cells. The advantages of organic polymers are that they are not only less expensive and more easily available than Si but also readily manufacturable into devices by solution-based processes [7]. In addition, the work function of organic polymers can be easily tuned in 
accordance with the combination of other materials [8,9]. For instance, the work function of poly(3,4-ethylenedioxythiophene) doped with a poly(styrenesulfonate) (PEDOT:PSS), which is known as a p-type-like conjugated conducting polymer that is also widely utilized as a hole transport layer, can be varied from 5.0 to $\sim 5.44 \mathrm{eV}$ by controlling the concentrations of the PSS dopant [8], other chemical dopants (e.g., glycerol and sorbitol) [9] and/or the thermal annealing conditions, such as temperature and atmospheric gases [9]. The work function of PEDOT:PSS can be adjusted so that it is closer to the valence band (VB) of Si, enabling the efficient delivery of holes in Si regions via a PEDOT:PSS layer. Therefore, the fabrication of inorganic/organic hybrid solar cells that combine $\mathrm{Si}$ with PEDOT:PSS [10] is an alternative to expensive Si-based solar cells, and they may promote the use of solar cells. To date, most research efforts have predominantly focused on the photovoltaic performance of Si/PEDOT:PSS hybrid solar cells [11-13]. However, the performance of such cells is inferior to that of Si-based solar cells. This is caused by the low generation rate of charge carriers in Si regions owing to high reflectance, although $\mathrm{Si}$ can absorb light over the broad wavelength range of the solar spectrum. The formation of micro-textured structures on the Si surface is a method for suppressing the reflectance and for improving the photovoltaic performance because they promote the scattering of incident light. In the fabrication of such inorganic/organic hybrid solar cells with micro-textured structures, controlling the PEDOT:PSS coating onto the micro-textured Si surface and investigating the effects of the generation, separation, and collection of charge carriers in the hybrid solar cells are of crucial importance.

In this work, we prepared Si/PEDOT:PSS hybrid solar cells with micro-pyramidal structures. We investigated the effects of PEDOT:PSS coverage and the rear electrode on the photovoltaic performance of the hybrid solar cells. Optimizing the PEDOT:PSS coating and the rear electrode materials allowed us to promote the favorable separation of photogenerated charge carriers at the Si/PEDOT:PSS interface regions and their adequate collection at the electrode. Our approach could increase the PCE up to $8.25 \%$.

\section{Experimental Methods}

\subsection{Preparation of Micro-Pyramidal Structures onto the Surface of Si Substrates}

One-side-polished $525-\mu \mathrm{m}$-thick n-type crystalline Si-(100) substrates with resistivities of 1-50 $\Omega$ $\mathrm{cm}$ and a $15 \mathrm{~mm} \times 15 \mathrm{~mm}$ cell area were used. The Si substrates were cleaned with acetone, ethanol, and deionized water for $10 \mathrm{~min}$ each, followed by cleansing with piranha solution consisting of sulfuric acid $\left(\mathrm{H}_{2} \mathrm{SO}_{4}\right)$ and hydrogen peroxide $\left(\mathrm{H}_{2} \mathrm{O}_{2}\right)(3: 1, v / v)$ for $10 \mathrm{~min}$, and then with a $\%$ hydrofluoric acid (HF) solution for $2 \mathrm{~min}$ to remove any organic compounds and any native oxides. These cleaning processes are of crucial importance because the presence of residue on the substrate is detrimental to the uniform formation of micro-pyramidal structures. Following the cleaning process, the micro-pyramidal structures were fabricated on the Si substrates by anisotropic chemical etching in an alkaline solution consisting of $4.2 \mathrm{wt} \%$ potassium hydroxide $(\mathrm{KOH})$ and $10 \mathrm{wt} \%$ pure etch TAD80 (Hayashi Pure Chemical Ind. Co., Tokyo, Japan) at $85{ }^{\circ} \mathrm{C}$ for $25 \mathrm{~min}$.

\subsection{Fabrication of Si/PEDOT:PSS Hybrid Solar Cells with Micro-Pyramidal Structures}

Schematic diagrams of the Si/PEDOT:PSS hybrid solar cells with micro-pyramidal structures and the molecular structure of PEDOT:PSS are illustrated in Figure 1. First, the Si substrate with the micro-pyramidal structures was cleaned with piranha solution for $50 \mathrm{~min}$, followed by cleaning with a $2 \% \mathrm{HF}$ solution for $2 \mathrm{~min}$. Immediately after the cleaning process, highly conductive PEDOT:PSS mixed with $5 \mathrm{wt} \%$ dimethyl sulfoxide (DMSO) and $0.1 \mathrm{wt} \%$ Zonyl ${ }^{\circledR}$ fluorosurfactant (Sigma-Aldrich, Tokyo, Japan) was spin-coated onto the micro-pyramidal surface for $120 \mathrm{~s}$ in an atmosphere environment to form the hybrid solar cells, followed by annealing at $140{ }^{\circ} \mathrm{C}$ for $30 \mathrm{~min}$ in an atmosphere environment to remove any residual water inside the PEDOT:PSS. To investigate the influence of PEDOT:PSS coverage on the photovoltaic performance of the hybrid solar cells, the spin speeds were varied from 
$1900 \mathrm{rpm}$ to 3800 and $7500 \mathrm{rpm}$. Additionally, to examine the collection efficiencies of the separated charge carriers at the Si/PEDOT:PSS interface regions, the rear electrode materials used were Ag and $\mathrm{Al}$ (Kojundo Chemical Laboratory Co., Saitama, Japan). Ag or Al rear electrodes (200-nm-thick) were deposited by radio frequency (RF) sputtering onto the back side of the substrate. A 200-nm-thick Ag finger-grid front electrode was formed on the surface of PEDOT:PSS layer by RF sputtering through a shadow mask. The width of each finger of the Ag grid electrode was $100 \mu \mathrm{m}$, and the spacing between fingers was $500 \mu \mathrm{m}$.

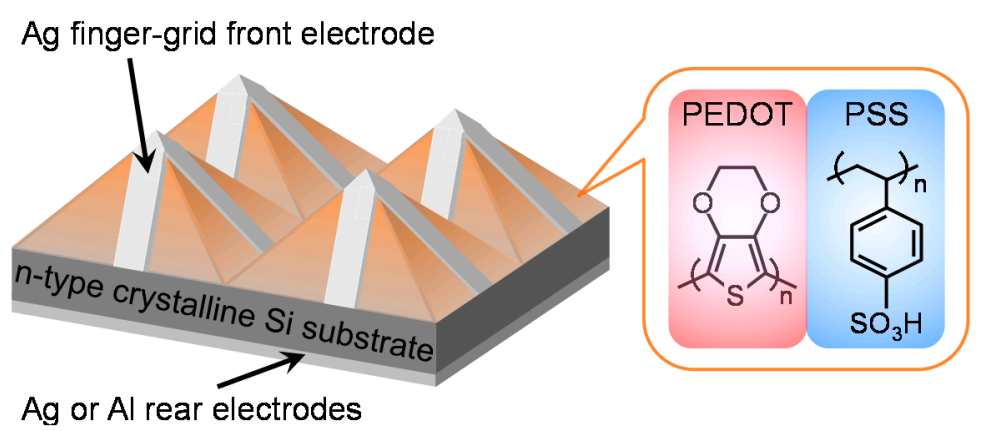

Figure 1. Schematic diagrams of the $\mathrm{Si} /$ poly(3,4-ethylenedioxythiophene):poly(styrenesulfonate) (PEDOT:PSS) hybrid solar cell with micro-pyramidal structures and the molecular structure of PEDOT:PSS.

\subsection{Characterization}

Scanning electron microscopy (SEM, JEOL Ltd., Tokyo, Japan) observations were used for morphology analysis of the micro-pyramidal-structured $\mathrm{Si}$ substrates and the micro-pyramidal-structured Si/PEDOT:PSS composites. Reflectance of the micro-pyramidal-structured Si substrates with and without PEDOT:PSS coverage were recorded by a V-770 ultraviolet/visible/near infrared (UV/VIS/NIR) spectrophotometer (JASCO Co., Tokyo, Japan) system coupled with an integrating sphere. The cell performance and current density-voltage characteristics of the micro-pyramidal-structured Si/PEDOT:PSS hybrid solar cells were measured at room temperature using a Keithley 2400 source meter (Keithley Instruments, Inc., Cleveland, OH, USA) under illumination of air mass (AM) $1.5 \mathrm{G} 100 \mathrm{~mW} / \mathrm{cm}^{2}$ simulated solar light, which was calibrated using a single-crystalline silicon solar cell (BS-500) provided by Sharp (Osaka, Japan). The series resistance $\left(R_{S}\right)$ and the shunt resistance $\left(R_{s h}\right)$ were estimated from the inverse of the slope of a tangent line in the range from $0 \mathrm{~V}$ to a point of peak power output $\left(P_{\max }\right)$ and from a point of $P_{\max }$ to $1 \mathrm{~V}$, respectively, in the current density-voltage curves by the method of least squares.

\section{Results and Discussion}

\subsection{Morphology and Reflectance Properties of Si/PEDOT:PSS Composites with Micro-Pyramidal Structures}

The morphology of the micro-pyramidal structures on the Si substrate surface was confirmed by tilted top-view and cross-sectional SEM micrographs, as shown in Figure 2a,b, respectively. The micro-pyramidal structures were found to be uniformly formed across the substrate surface. We confirmed that such micro-pyramids were heterogeneously distributed on the substrate surface when only the $\mathrm{KOH}$ solution was used. The good arrangement of the micro-pyramids is attributable to the pure etch TAD80 mixed with $\mathrm{KOH}$ solution. The pure etch TAD80 acts as a secondary dopant to attach the micro-mask onto the Si substrate surface and to dissociate bubbles on the sample surface, which happens during alkaline etching of the Si layer. These behaviors facilitated the adequate alkaline etching of the Si layer according to the (111) crystal orientation corresponding to facets of the micro-pyramids, thereby enabling the formation of high-density textured pyramids. The average width and average height of the obtained micro-pyramids were $0.8 \mu \mathrm{m}$ and $1 \mu \mathrm{m}$, respectively. 


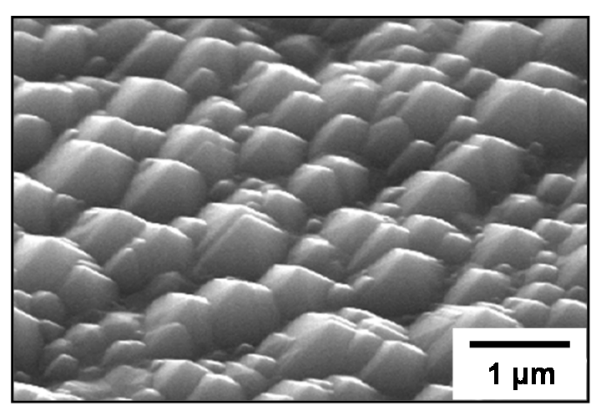

(a)

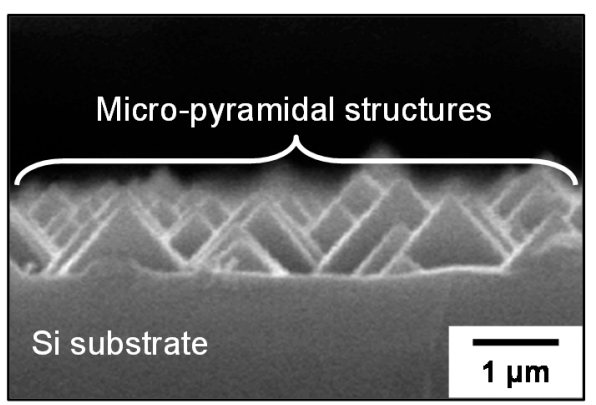

(b)

Figure 2. (a) Tilted top-view; and (b) cross-sectional Scanning electron microscopy (SEM) images of micro-pyramidal structures on the surface of the Si substrate.

The morphologies of the PEDOT:PSS layers on the micro-pyramidal surfaces fabricated at spin speeds of 1900, 3800, and $7500 \mathrm{rpm}$ were observed by the cross-sectional SEM micrographs, shown in Figure 3a-c, respectively. The PEDOT:PSS layers show smooth surfaces without any cracks for all spin speeds. However, the spin speed strongly affected the adhesion and thickness of the PEDOT:PSS layer. At $1900 \mathrm{rpm}$, it was very difficult for the PEDOT:PSS to penetrate into the narrow valleys of the micro-pyramids during spin coating, although the PEDOT:PSS layer could be coated on the micro-pyramidal surfaces. In the case of the low spin speed, the PEDOT:PSS cured around the upper parts of the micro-pyramidal surfaces. As a result, voids formed in valleys of the micro-pyramids, as shown in Figure 3a. Increasing the spin speed to $3800 \mathrm{rpm}$ improved the penetration of PEDOT:PSS into the valleys of the micro-pyramids because of the increase in both centrifugal force and capillary force, resulting in a reduction in the number of voids, as shown in Figure 3b. At $7500 \mathrm{rpm}$, the voids completely disappeared, as shown in Figure 3c. The perfect adhesion of the PEDOT:PSS layer throughout the micro-pyramidal surfaces is mainly due to the enhanced penetration rate of PEDOT:PSS rather than to the hardening rate by the increased centrifugal and capillary forces. Additionally, these spin speeds also affected the thickness of the PEDOT:PSS layer on the micro-pyramidal surfaces. The average thickness ( $T_{\text {ave }}$ ) of the PEDOT:PSS layer decreased from approximately $35 \mathrm{~nm}$ to approximately $25 \mathrm{~nm}$ with increasing spin speed from $1900 \mathrm{rpm}$ to $7500 \mathrm{rpm}$. The thickness of the PEDOT:PSS layer plays a significant role in the efficiency of exciton diffusion to a $\mathrm{p} / \mathrm{n}$ junction interface, corresponding to the micro-pyramidal-structured Si/PEDOT:PSS. The efficiency of exciton diffusion, which causes the generation of charge carriers in the PEDOT:PSS regions, is closely related to exciton diffusion lengths $\left(L_{D}\right)$ [14]. The $L_{D}$ is usually a value of less than $20 \mathrm{~nm}$ for conjugated conducting polymers [14,15]. From the value of the $L_{D}$, the PEDOT:PSS coverage fabricated at $7500 \mathrm{rpm}$ enable the efficient generation of charge carriers owing to the favorable exciton diffusion. Further reduction in the thickness of the PEDOT:PSS layer might be facilitated by the generation of charge carriers. However, it was very difficult for the spin coater used in this study to obtain the thickness of less than $25 \mathrm{~nm}$ because the maximum spin speed of the spin coater was $7500 \mathrm{rpm}$. Most importantly, the $\mathrm{p} / \mathrm{n}$ junction areas, which result in the adequate separation of the photo-generated charge carriers, can be expanded by improving the adhesiveness of the PEDOT:PSS coverage on the micro-pyramidal surfaces. This indicates that it is important to control the spin speed so as to improve the adhesion of the PEDOT:PSS layer across and between the micro-pyramidal surfaces. It was, thus, found that the most suitable condition for PEDOT:PSS coverage onto the micro-pyramidal surfaces was the spin speed of $7500 \mathrm{rpm}$ and its corresponding thickness of approximately $25 \mathrm{~nm}$. 


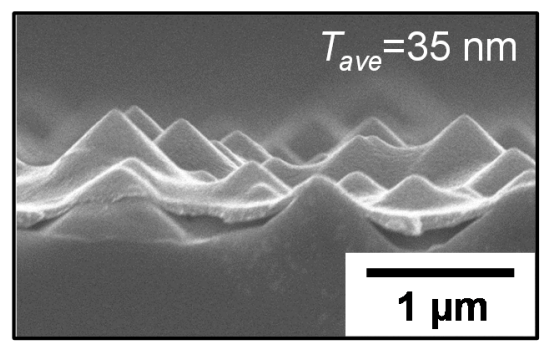

(a)

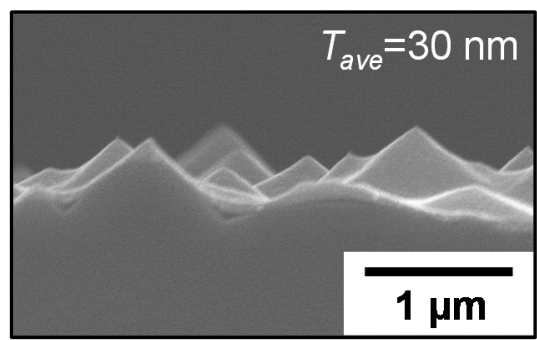

(b)

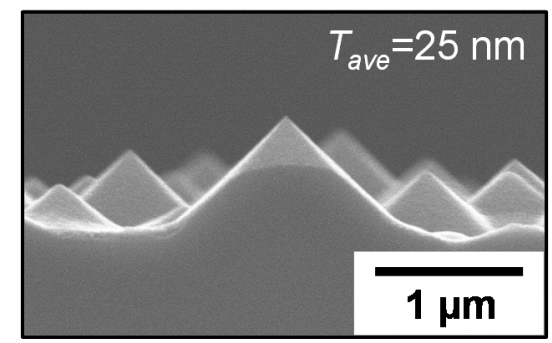

(c)

Figure 3. Cross-sectional SEM images of PEDOT:PSS layers on the micro-pyramidal surfaces coated with spin speeds of (a) 1900; (b) 3800; and (c) $7500 \mathrm{rpm}$.

To demonstrate the antireflection effect of the micro-pyramidal structures on the Si substrate surface, reflectance analysis was conducted, and the results are shown in Figure 4a. For the purpose of comparison, the same experiments were also carried out for a planar Si substrate. It can be seen that the planar Si substrate exhibit a strong reflectance of more than $70 \%$ in the incident wavelength range above $1100 \mathrm{~nm}$, corresponding to the optical bandgap of Si. However, the reflectance decreased to below $40 \%$ in the incident wavelength range $500-1000 \mathrm{~nm}$. As we expected, the reflectance decreased further by the introduction of the micro-pyramidal structures [16], resulting in less than $28 \%$ reflectance in the 500-1000 nm wavelength range. This decrease arose from the increased effective optical path length of incident light in the micro-pyramidal structures due to light trapping and light scattering, proving the effectiveness of the antireflection effect. The antireflection effect was further promoted by the PEDOT:PSS coverage onto the micro-pyramidal surfaces. The reflectance properties of the micro-pyramidal structures with and without PEDOT:PSS layer are shown in Figure 4b. The reflectance spectrum (black line) of micro-pyramidal structures before PEDOT:PSS coverage in Figure $4 \mathrm{~b}$ is identical to the reflectance spectrum (red line) of micro-pyramidal structures in Figure 4a. Interestingly, the micro-pyramidal surfaces with the PEDOT:PSS coating exhibited a remarkably low reflectance below $13 \%$ in the incident wavelength range of $500-1000 \mathrm{~nm}$. The stronger suppression of reflectance was achieved through the support of the PEDOT:PSS layer, which has absorption regions at ultraviolet (UV) wavelengths and near-infrared (IR) wavelengths. The PSS component shows intense absorbance in the UV region (an absorption peak at approximately $225 \mathrm{~nm}$ ) [17] and the PEDOT component exhibits broad absorbance in the near-IR region (an absorption peak at approximately 800-3000 nm) [18]. Therefore, this result demonstrates that the combination of micro-pyramidal structures and PEDOT:PSS coating significantly enhance the antireflection capability of the Si substrate surface. 


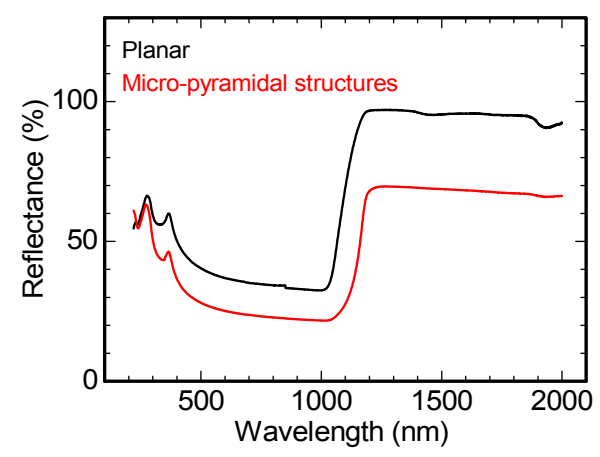

(a)

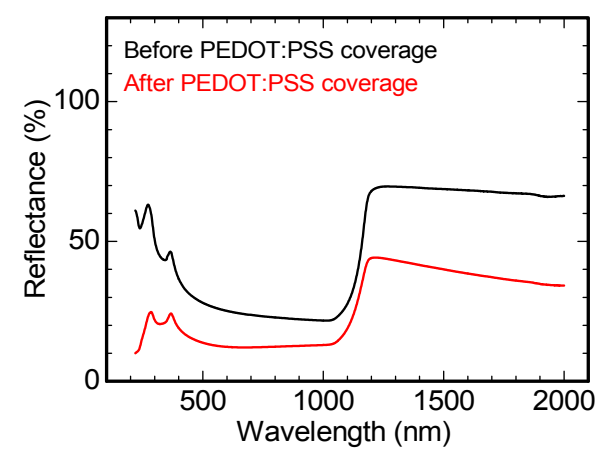

(b)

Figure 4. Reflectance spectra of (a) planar Si substrate and micro-pyramidal structures on the Si substrate surface; and (b) micro-pyramidal structures before and after PEDOT:PSS coverage. The micro-pyramidal-structured Si/PEDOT:PSS composites in (b) were fabricated by coating the PEDOT:PSS at 7500 rpm.

\subsection{Effect of PEDOT:PSS Coverage of Si/PEDOT:PSS Hybrid Solar Cells with Micro-Pyramidal Structures}

To clarify the effect of PEDOT:PSS coverage on the photovoltaic performance of micro-pyramidal-structured Si/PEDOT:PSS hybrid solar cells with a Ag rear electrode, current density-voltage characteristics were measured under $100 \mathrm{~mW} / \mathrm{cm}^{2}$ air mass $1.5 \mathrm{G}$ solar illumination, and the results are shown in Figure 5. The photovoltaic performance was also compared to that of a planar Si/PEDOT:PSS hybrid solar cell. Their corresponding photovoltaic parameters-short-circuit current density $\left(J_{s c}\right)$, open-circuit voltage $\left(V_{o c}\right)$, fill factor $(\mathrm{FF})$, and PCE-are summarized in Table 1. The planar Si/PEDOT:PSS hybrid solar cell showed poor performance, with a $J_{s c}$ of $19.9 \mathrm{~mA} / \mathrm{cm}^{2}$, a $V_{o c}$ of $0.308 \mathrm{~V}$, an FF of 0.282 , and a PCE of $1.72 \%$. The hybrid solar cell possessed not only high reflectance over the broad spectrum of 500-1000 nm, as identified by the reflectance analysis in Figure 4a, but also reduced junction areas of the $\mathrm{p} / \mathrm{n}$ interface corresponding to the $\mathrm{Si} / \mathrm{PEDOT}$ PSS. In such cases, the generation of charge carriers in the Si/PEDOT:PSS regions and their separation at the $\mathrm{p} / \mathrm{n}$ junction interface was inefficient. In particular, the separation rate of the photogenerated charge carriers affected the $J_{s c}$. Excitons in the conjugated conducting polymers are known to have binding energies in the range of 200-400 meV [15], which are sufficiently larger than the Si with excitonic binding energies of 10-15 meV [19-21]. Due to the larger excitonic binding energy in conjugated conducting polymers, exciton dissociation into holes and electrons at the $\mathrm{p} / \mathrm{n}$ junction interface, that is, electron transfer from the PEDOT:PSS regions to the Si regions, is caused by the internal electric fields built by Si/PEDOT:PSS and by the energy difference $(550 \mathrm{meV})$ between the lowest unoccupied molecular orbital (LUMO) energy level of the p-type conjugated conducting polymer such as PEDOT:PSS and the conduction band (CB) of $\mathrm{Si}[14,15]$. Similarly, exciton dissociation in the $\mathrm{Si}$ at the $\mathrm{p} / \mathrm{n}$ junction interface, that is, hole transfer from the Si regions to the PEDOT:PSS regions, is caused by the internal electric fields built by $\mathrm{Si}$ / PEDOT:PSS and by the energy difference $(70 \mathrm{meV})$ between the highest occupied molecular orbital (HOMO) energy level of PEDOT:PSS and the VB of Si. In the planar Si/PEDOT:PSS hybrid solar cell, the numbers of the separated electrons and holes were considerably poor due to the reduced $\mathrm{p} / \mathrm{n}$ junction areas. As a result, the hybrid solar cell showed the lowest $J_{s c}$ owing to the low collection rate of the separated charge carriers at each electrode. Additionally, the hybrid solar cell had a high $R_{S}$ of $3.22 \Omega \cdot \mathrm{cm}^{2}$ and a low $R_{s h}$ of $1.94 \times 10 \Omega \cdot \mathrm{cm}^{2}$, thereby leading to the low FF. Because of the possession of low $J_{s c}$ and FF, the planar Si/PEDOT:PSS hybrid solar cell exhibited poor PCE. 


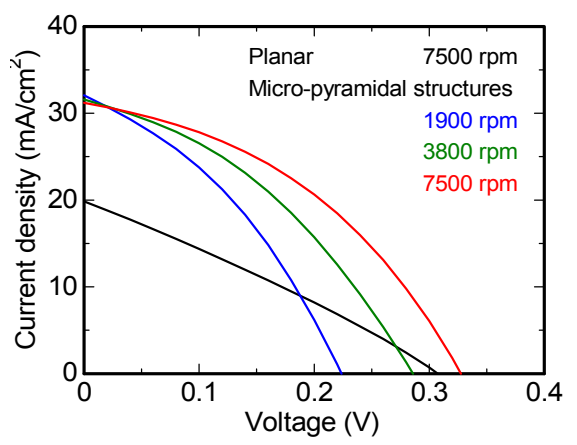

Figure 5. Current density-voltage characteristics of a planar Si/PEDOT:PSS hybrid solar cell fabricated at $7500 \mathrm{rpm}$ and the micro-pyramidal structured Si/PEDOT:PSS hybrid solar cells fabricated at various spin speeds. All of the hybrid solar cells used an Ag rear electrode.

Table 1. Photovoltaic parameters of planar Si/PEDOT:PSS hybrid solar cell and micro-pyramidal structured Si/PEDOT:PSS hybrid solar cells. All of the hybrid solar cells used an Ag rear electrode. FF: fill factor; and PCE: high power conversion efficiency.

\begin{tabular}{cccccc}
\hline Device & Spin Speed $(\mathbf{r p m})$ & $J_{\text {sc }}\left(\mathbf{m A} / \mathbf{c m}^{\mathbf{2}}\right)$ & $V_{\boldsymbol{o c}}(\mathrm{V})$ & FF & PCE (\%) \\
\hline Planar & 7500 & 19.9 & 0.308 & 0.282 & 1.72 \\
Micro-pyramidal structure & 1900 & 32.1 & 0.224 & 0.360 & 2.58 \\
Micro-pyramidal structure & 3800 & 31.6 & 0.286 & 0.372 & 3.36 \\
Micro-pyramidal structure & 7500 & 31.2 & 0.328 & 0.403 & 4.13 \\
\hline
\end{tabular}

The poor cell performance was dramatically improved by introducing the micro-pyramidal structures in the hybrid solar cells. The hybrid solar cells that combined the micro-pyramidal structures with PEDOT:PSS showed remarkably low reflectance, as shown in Figure $4 \mathrm{~b}$. These hybrid solar cells enabled the efficient generation of charge carriers in the micro-pyramidal-structured Si/PEDOT:PSS regions. Intriguingly, there was almost no variation of $J_{s c}$ between the hybrid solar cells fabricated at different spin speeds. The reason for this phenomenon is as follows. The hybrid solar cell fabricated at $1900 \mathrm{rpm}$ formed voids in the valleys of the micro-pyramids because of deficient PEDOT:PSS coverage, as identified by SEM analysis in Figure 3a. In the hybrid solar cell with the voids, the photogenerated charge carriers separate only at the $\mathrm{p} / \mathrm{n}$ junction interface around the top and middle parts of the micro-pyramids, where the PEDOT:PSS layer is in immediate contact with the Si layer. The separated electrons and holes are transferred to the Si regions and PEDOT:PSS regions, respectively, so as to travel to each electrode. The separated electrons also provoke the recombination with holes during transport to the rear electrode. Thus, the hybrid solar cell fabricated at $1900 \mathrm{rpm}$ showed a $J_{s c}$ of $32.1 \mathrm{~mA} / \mathrm{cm}^{2}$, as shown in Figure 5 and Table 1, despite the decreased collection rate of the separated charge carriers at each electrode owing to the restricted carrier separation and the occurrence of carrier recombination. Most importantly, the production volume of voids can be reduced by increasing the spin speed. As a matter of fact, the spin speed of $3800 \mathrm{rpm}$ decreased the number of voids, as shown in Figure $3 \mathrm{~b}$. The reduced number of voids served to expand the $\mathrm{p} / \mathrm{n}$ junction areas, resulting in efficient carrier separation. In other words, carrier separation also occurred in the valleys of the micro-pyramids. As a result, hole recombination increased during transport of the charge carriers to the rear electrode because the separation rate of the photogenerated charge carriers was enhanced. Owing to the increased recombination probability of separated charge carriers, the hybrid solar cell fabricated at $3800 \mathrm{rpm}$ exhibited a $J_{s c}$ of $31.6 \mathrm{~mA} / \mathrm{cm}^{2}$, as shown in Figure 5 and Table 1 . As the spin speed was further increased from $3800 \mathrm{rpm}$ to $7500 \mathrm{rpm}$, the adhesiveness of the PEDOT:PSS coverage on the micro-pyramidal surfaces dramatically improved, as shown in Figure 3c, leading to the disappearance of voids. The perfect adhesion of the PEDOT:PSS layer throughout the micro-pyramidal surfaces further increased the separation rate of the photogenerated charge carriers, thereby causing 
a higher recombination rate. Therefore, the $J_{s c}$ value of the hybrid solar cell fabricated at $7500 \mathrm{rpm}$, $31.2 \mathrm{~mA} / \mathrm{cm}^{2}$, was roughly equal to the value of the hybrid solar cell fabricated at $3800 \mathrm{rpm}$, as shown in Figure 5 and Table 1. These results imply that the PCE may be determined by other photovoltaic factors such as $V_{o c}$ and FF for our micro-pyramidal-structured Si/PEDOT:PSS hybrid solar cells.

The hybrid solar cell fabricated at $1900 \mathrm{rpm}$ showed a $V_{o c}$ of $0.224 \mathrm{~V}$ and an FF of 0.360 , as exhibited in Figure 5 and Table 1. These photovoltaic parameters were very poor as compared to those of the hybrid solar cells fabricated at higher spin speeds because of the presence of voids in the valleys of the micro-pyramids after PEDOT:PSS coating. The formation of voids in the hybrid solar cell led to a high $R_{S}$ of $6.03 \times 10^{-1} \Omega \cdot \mathrm{cm}^{2}$ and a low $R_{s h}$ of $1.58 \times 10 \Omega \cdot \mathrm{cm}^{2}$ as compared to the values at higher spin speeds, resulting in lower $V_{o c}$ and FF. Yang, et al. reported that voids are likely to form local shunts, which cause the deterioration of $V_{o c}$ and FF [22]. This trend is in accord with our results. Due to the degradation of $V_{o c}$ and FF by inhomogeneous PEDOT:PSS coating, the hybrid solar cell fabricated at $1900 \mathrm{rpm}$ exhibited a poor PCE of $2.58 \%$, as shown in Table 1 . However, when the spin speed was increased from $1900 \mathrm{rpm}$ to $3800 \mathrm{rpm}$, the $V_{o c}$ and FF of the hybrid solar cell increased to $0.286 \mathrm{~V}$ and 0.372 , respectively, as shown in Figure 5 and Table 1 . Additionally, the hybrid solar cell fabricated at $3800 \mathrm{rpm}$ also had a lower $R_{S}$ of $5.06 \times 10^{-1} \Omega \cdot \mathrm{cm}^{2}$ and a higher $R_{s h}$ of $2.63 \times 10 \Omega \cdot \mathrm{cm}^{2}$, thereby leading to higher $V_{o c}$ and FF values. These results may be related to the adhesiveness of the PEDOT:PSS layer on the micro-pyramidal surfaces. Therefore, the PCE of the hybrid solar cell fabricated at $3800 \mathrm{rpm}$ was enhanced to $3.36 \%$ by increasing the adhesion areas of the PEDOT:PSS layer and by the increasing $V_{o c}$ and FF, as shown in Table 1 . At $7500 \mathrm{rpm}$, the hybrid solar cell exhibited good photovoltaic parameters, with a $V_{o c}$ of $0.328 \mathrm{~V}$ and an FF of 0.403 , as shown in Figure 5 and Table 1. The increase in $V_{o c}$ and FF is inferred from the subequal $R_{S}$ value of $5.44 \times 10^{-1} \Omega \cdot \mathrm{cm}^{2}$ and the increase in $R_{s h}$ to $3.84 \times 10 \Omega \cdot \mathrm{cm}^{2}$. These results are due to the complete adhesion of the PEDOT:PSS layer throughout the micro-pyramidal surface as a result of the high spin speed. Thus, the hybrid solar cell fabricated at $7500 \mathrm{rpm}$ attained a high PCE of $4.13 \%$ by improving the $V_{o c}$ and FF and the adhesiveness of the PEDOT:PSS coverage, as shown in Table 1. Unfortunately, the $V_{o c}$ of these hybrid solar cells showed relatively low values as compared to the hybrid solar cells fabricated by other research groups, although the $V_{o c}$ enhanced with increasing $R_{s h}$ owing to the good adhesiveness of the PEDOT:PSS layer causing the disappearance of voids. Chen et al. reported that the micro-pyramidal-structured Si/PEDOT:PSS hybrid solar cells using the Al rear electrode show an upward trend of the $V_{o c}$ from $0.31 \mathrm{~V}$ to $0.52 \mathrm{~V}$ for spin speed from $1000 \mathrm{rpm}$ to $8000 \mathrm{rpm}$ [16]. The main reason of the lower $V_{o c}$ of our hybrid solar cells using an Ag rear electrode is as follows. The $V_{o c}$ value is strongly affected by the work function of the metal, as we will discuss in detail in a later section. Liu et al. reported that the $V_{o c}$ is determined by the energy difference between the HOMO energy level of the p-type conjugated conducting polymer such as PEDOT:PSS and the Fermi level of the metal when the value of the work function is larger than the electron affinity of Si [23]. The hybrid solar cells using the $\mathrm{Ag}$ rear electrode possess the electron affinity of $\mathrm{Si}$ of $4.05 \mathrm{eV}$ and the work function of $\mathrm{Ag}$ of 4.6-4.8 eV [24-27], as depicted in the energy band models of Figure 6b; the theoretical values of $V_{o c}$ are $0.3-0.5 \mathrm{eV}$. Therefore, the lower $V_{o c}$ of the hybrid solar cells using the Ag rear electrode is attributable to the usage of $\mathrm{Ag}$ with the larger work function than the electron affinity of Si. The abovementioned results clearly suggest that it is important to optimize the adhesiveness of PEDOT:PSS coverage on the micro-pyramidal surfaces and also to inhibit carrier recombination in order to improve the PCE of the micro-pyramidal-structured Si/PEDOT:PSS hybrid solar cells. Thus, the optimal spin speed for good adhesiveness of the PEDOT:PSS layer was $7500 \mathrm{rpm}$. 


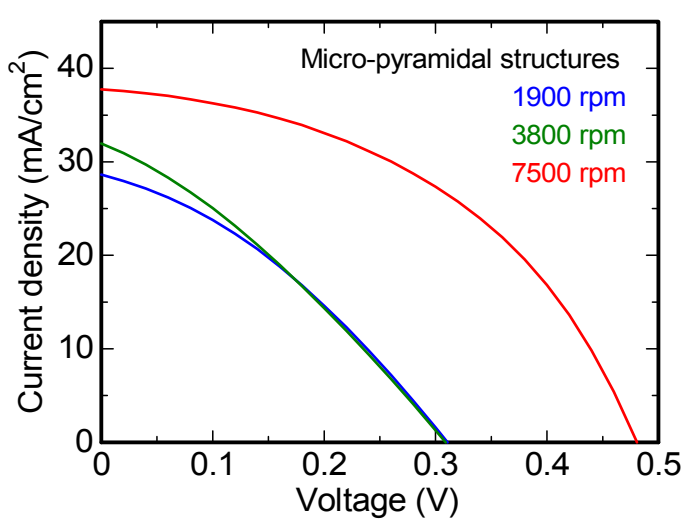

(a)

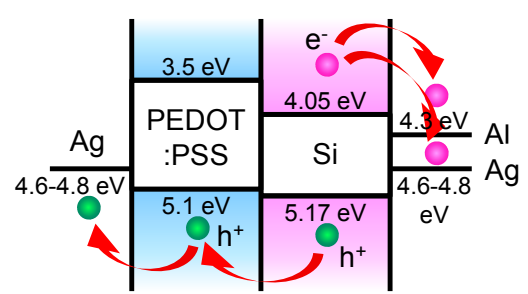

(b)

Figure 6. (a) Current density-voltage characteristics of micro-pyramidal-structured Si/PEDOT:PSS hybrid solar cells fabricated at various spin speeds. All of the hybrid solar cells used Al rear electrodes; and (b) energy band diagrams of micro-pyramidal-structured Si/PEDOT:PSS hybrid solar cells using Al or Ag rear electrodes. The arrows indicate the transfer processes of the photogenerated charge carriers.

\subsection{Effect of Rear Electrode of Si/PEDOT:PSS Hybrid Solar Cells with Micro-Pyramidal Structures}

The effect of the rear electrode on the photovoltaic performance of the micro-pyramidal-structured $\mathrm{Si} / \mathrm{PEDOT}$ PSS hybrid solar cells in order to further improve the PCE was also investigated. The best PCE among the hybrid solar cells using an Ag rear electrode was $4.13 \%$ because of the recombination of separated electrons with holes during transport to the rear electrode, as identified by the cell performance analysis shown in Figure 5. Additionally, the hybrid solar cells did not show an increase in $J_{s c}$, despite the improved adhesiveness of PEDOT:PSS layer on the micro-pyramidal surfaces. To increase the collection rate of separated electrons, we used $\mathrm{Al}$ as the rear electrode. The current density-voltage characteristics of the micro-pyramidal-structured Si/PEDOT:PSS hybrid solar cells using an $\mathrm{Al}$ rear electrode were measured under $100 \mathrm{~mW} / \mathrm{cm}^{2} \mathrm{AM} 1.5 \mathrm{G}$ solar illumination, and the results are shown in Figure 6a. The photovoltaic parameters of the hybrid solar cells using the $\mathrm{Al}$ rear electrode are summarized in Table 2 . As we expected, the $J_{s c}$ of the hybrid solar cells showed an upward trend with increasing spin speed, increasing from $28.6 \mathrm{~mA} / \mathrm{cm}^{2}$ to $37.8 \mathrm{~mA} / \mathrm{cm}^{2}$ as the spin speed increased from $1900 \mathrm{rpm}$ to $7500 \mathrm{rpm}$, as shown in Figure 6a and Table 2. This is obviously different from the $J_{s c}$ of the hybrid solar cells using an Ag rear electrode, as shown in Figure 5 and Table 1. This is mainly due to the difference in the collection process of separated electrons at rear electrode because $\mathrm{Al}$ and $\mathrm{Ag}$ have different work functions [24-28]. The collection process of separated electrons can be described by means of energy band models, as shown in Figure 6b. The energy level of the $\mathrm{Al}$ electrode is located close to the $\mathrm{CB}$ of $\mathrm{Si}$, corresponding to the electron affinity of $\mathrm{Si}$ because the work function of $\mathrm{Al}$ is $4.3 \mathrm{eV}[25,28]$. In contrast, the energy level of the Ag electrode is located close to the VB of Si because the work function of Ag is approx. 4.6-4.8 eV [24-27]. Due to these different energies levels at the $\mathrm{Al}$ and $\mathrm{Ag}$ electrodes, the collection process of separated electrons at each rear electrode is different. The separated holes at the $\mathrm{p} / \mathrm{n}$ junction interface, corresponding to the micro-pyramidal-structured Si/PEDOT:PSS, can be efficiently collected at the Ag finger-grid front electrode via the PEDOT:PSS regions because the energy level of Ag is located close to the HOMO energy level of PEDOT:PSS, as shown in Figure $6 \mathrm{~b}$. In contrast, the separated electrons at the $\mathrm{p} / \mathrm{n}$ junction interface can be collected by the different processes for each rear electrode. Regarding the collection process of separated electrons, Thiyagu et al. [29] reported that the holes, which are minority carriers in n-type $\mathrm{Si}$, recombine with separated electrons via trap levels at the interface regions of the Si back surface/rear electrode. They also suggested that the carrier recombination at the Si back surface/rear electrode interface is suppressed by forming back-surface field (BSF) regions consisting of a heavily doped $\mathrm{n}^{+}$layer because the BSF regions act as barrier layer to prevent the ingress of 
holes [29]. Our hybrid solar cells did not form BSF regions at the Si back surface/rear electrode interface. In the case of the hybrid solar cells using the Ag rear electrode, the holes in Si regions may be transferred not only toward the PEDOT:PSS regions, but also toward the Ag rear electrode because of the small energy difference between the energy level of Ag and the VB of $\mathrm{Si}$, as shown in Figure 6b. This easily causes the recombination of holes with separated electrons at the Si back surface/rear electrode interface, as discussed in the previous section. The carrier recombination is also generated via trap levels at the interface regions, as was suggested by Thiyagu et al. [29]. These phenomena become active with increasing spin speeds because the increased spin speeds expand the $\mathrm{p} / \mathrm{n}$ junction areas, which result in efficient carrier separation. Therefore, the hybrid solar cells using the Ag rear electrode did not demonstrate increasing $J_{s c}$ with increasing spin speeds because the collection rate of the separated electrons at the Ag rear electrode remained unchanged owing to the enhancement of both the separation rate and the recombination probability of the photogenerated charge carriers.

Table 2. Photovoltaic parameters of micro-pyramidal-structured Si/PEDOT:PSS hybrid solar cells. All of the hybrid solar cells used Al rear electrodes.

\begin{tabular}{cccccc}
\hline Device & Spin Speed $(\mathbf{r p m})$ & $J_{\text {sc }}\left(\mathbf{m A} / \mathbf{c m}^{2}\right)$ & $V_{\text {oc }}(\mathbf{V})$ & FF & PCE (\%) \\
\hline Micro-pyramidal structure & 1900 & 28.6 & 0.311 & 0.341 & 3.03 \\
Micro-pyramidal structure & 3800 & 32.0 & 0.309 & 0.308 & 3.04 \\
Micro-pyramidal structure & 7500 & 37.8 & 0.481 & 0.454 & 8.25 \\
\hline
\end{tabular}

On the other hand, as the $\mathrm{Ag}$ rear electrode is replaced with $\mathrm{Al}$, the energy level of the metal changes; that is, the energy level of $\mathrm{Al}$ is relocated close to the $\mathrm{CB}$ of $\mathrm{Si}$, as shown in Figure $6 \mathrm{~b}$. In this case, the transfer of holes from the $\mathrm{Si}$ regions toward the $\mathrm{Al}$ rear electrode is inhibited because of the larger energy difference between the energy level of $\mathrm{Al}$ and the $\mathrm{VB}$ of $\mathrm{Si}$, as shown in Figure $6 \mathrm{~b}$. As a result, the hybrid solar cells using the $\mathrm{Al}$ rear electrode show decreased carrier recombination at the $\mathrm{Si}$ back surface/rear electrode interface as compared to the hybrid solar cells using the Ag rear electrode, although carrier recombination via trap levels at the interface regions is generated. The reduction of the recombination probability of separated charge carriers increases the collection rate of separated electrons at the $\mathrm{Al}$ rear electrode. In hybrid solar cells using the $\mathrm{Al}$ rear electrode, the holes in the $\mathrm{Si}$ regions are efficiently transferred toward the PEDOT:PSS regions owing to the slight energy difference between the VB of Si and the HOMO energy level of PEDOT:PSS, as shown in Figure 6b. Additionally, the separated electrons in the Si regions are easily transferred toward the $\mathrm{Al}$ rear electrode owing to the small energy difference between the CB of $\mathrm{Si}$ and the energy level of $\mathrm{Al}$, as shown in Figure $6 \mathrm{~b}$, resulting in the effective collection of separated electrons at the $\mathrm{Al}$ rear electrode. The collection rate of the separated electrons can be further enhanced by increasing the spin speeds. This arises from the increase in the separation rate of photogenerated charge carriers by the resolution of the adhesiveness of the PEDOT:PSS layer on the micro-pyramidal surfaces for the hybrid solar cells fabricated at spin speeds of more than $3800 \mathrm{rpm}$. Thus, the hybrid solar cells using an $\mathrm{Al}$ rear electrode attained a high $J_{s c}$ of $37.8 \mathrm{~mA} / \mathrm{cm}^{2}$ at $7500 \mathrm{rpm}$, as shown in Figure 6a and Table 2.

It is particularly worth noting that the hybrid solar cells using the $\mathrm{Al}$ rear electrode showed other dramatically enhanced photovoltaic factors such as $V_{o c}$ and FF. Indeed, the $V_{o c}$ of the hybrid solar cells using the $\mathrm{Al}$ rear electrode increased from $0.311 \mathrm{~V}$ to $0.481 \mathrm{~V}$ with increasing spin speed from $1900 \mathrm{rpm}$ to $7500 \mathrm{rpm}$, as shown in Figure 6a and Table 2. The enhancement of $V_{o c}$ is related to the increase in $R_{s h}$ [14,30]; the increased $R_{s h}$ resulted in low power losses in the hybrid solar cells under the equivalent circuit model $[14,30]$. The hybrid solar cell fabricated at $7500 \mathrm{rpm}$ had a high $R_{s h}$ of $8.06 \times 10 \Omega \cdot \mathrm{cm}^{2}$ as compared to the hybrid solar cell fabricated at $1900 \mathrm{rpm}$, which had an $R_{s h}$ of $3.09 \times 10 \Omega \cdot \mathrm{cm}^{2}$. Because of the increased $R_{s h}$, the hybrid solar cell fabricated at $7500 \mathrm{rpm}$ achieved a high $V_{o c}$. Such a trend is consistent with the variation in the hybrid solar cells using the Ag rear electrode. More noteworthy is the fact that the $V_{o c}$ of the hybrid solar cells using the Al rear electrode was substantially increased as compared to the hybrid solar cells using Ag rear electrode, whose $V_{o c}$ 
increased from $0.224 \mathrm{~V}$ to $0.328 \mathrm{~V}$ for spin speeds from $1900 \mathrm{rpm}$ to $7500 \mathrm{rpm}$, as shown in Figure 5 and Table 1. The substantial increase in $V_{o c}$ can be described by means of energy band models in Figure $6 \mathrm{~b}$. In the hybrid solar cells consisting of the micro-pyramidal-structured Si/PEDOT:PSS, the $V_{o c}$ value is based on the correlation between the electron affinity of Si and the work function of the metal that is used as the rear electrode. When the value of the work function is larger than the electron affinity of $\mathrm{Si}$, the $V_{o c}$ is strongly affected by the work function of the metal, as suggested by Liu et al. [23]. They also reported that $V_{o c}$ is determined by the energy difference between the HOMO energy level of the p-type conjugated conducting polymer such as PEDOT:PSS and the Fermi level of the metal [23]. In our hybrid solar cells, the electron affinity of $\mathrm{Si}$ is $4.05 \mathrm{eV}$, and the work functions of $\mathrm{Al}$ and $\mathrm{Ag}$ are $4.3 \mathrm{eV}[25,28]$ and 4.6-4.8 eV [24-27], respectively. In such cases, the theoretical values of $V_{o c}$ of the hybrid solar cells using the $\mathrm{Ag}$ rear electrode are estimated to be $0.3-0.5 \mathrm{eV}$. These $V_{o c}$ values are similar to the measured values of the hybrid solar cells using the Ag rear electrode. The difference between the theoretical values and the measured values of $V_{o c}$ is attributable to the low $R_{s h}$ value of $3.84 \times 10 \Omega \cdot \mathrm{cm}^{2}$, which strongly depends on the adhesiveness of the PEDOT:PSS layer on the micro-pyramidal surfaces. Therefore, the lower $V_{o c}$ of the hybrid solar cells using Ag rear electrode is mainly due to the usage of $\mathrm{Ag}$, whose work function is sufficiently larger than the electron affinity of $\mathrm{Si}$. On the other hand, the theoretical value of $V_{o c}$ of the hybrid solar cells using the $\mathrm{Al}$ rear electrode was estimated to be $0.8 \mathrm{eV}$. This implies that the reduced energy difference between the work function and the electron affinity of Si can result in an increase in $V_{o c}$. Thus, the hybrid solar cells using the $\mathrm{Al}$ rear electrode obtained a high $V_{o c}$ of $0.481 \mathrm{~V}$ for the highest spin speed of $7500 \mathrm{rpm}$, even though there are significant differences between the theoretical values and the measured values. Additionally, the hybrid solar cell showed a significant improvement in the FF to 0.454 , as shown in Table 2 . The enhancement in the FF is mainly derived from the decrease in $R_{S}$ of the hybrid solar cell fabricated at $7500 \mathrm{rpm}$ to $1.07 \Omega \cdot \mathrm{cm}^{2}$; the $R_{S}$ value of the hybrid solar cell fabricated at $1900 \mathrm{rpm}$ was $2.47 \Omega \cdot \mathrm{cm}^{2}$. Thus, the Al rear electrode used in the hybrid solar cells improved $V_{o c}$ and FF, in addition to $J_{s c}$. Due to the considerable improvement of these photovoltaic parameters, the hybrid solar cell fabricated at $7500 \mathrm{rpm}$ exhibited the best PCE of $8.25 \%$, as shown in Table 2. The abovementioned results clearly suggest that it is crucially important to adopt a rear electrode material that enables the efficient collection of separated electrons in order to further improve the PCE of the micro-pyramidal-structured $\mathrm{Si} / \mathrm{PEDOT}$ :PSS hybrid solar cells. It is also demonstrated that the correlation between the electron affinity of $\mathrm{Si}$ and the work function of the metal is an important indicator for improving the photovoltaic parameters. Consequently, the optimal rear electrode material leading to the best PCE was Al, as specified in this section. Additionally, other factors, such as the stability, may be also taken into account. The stability of $\mathrm{Al}$ is poor as compared to $\mathrm{Ag}$ because $\mathrm{Al}$ is easily oxidized in an atmosphere environment owing to the high ionization tendency. For instance, $\mathrm{In} / \mathrm{Ga}$ alloys with a work function of 4.08-4.25 eV [31-34], which is similar to work function of Al, might be effective as an alternative to $\mathrm{Al}$ because they have low ionization tendencies as compared to Al. Regarding the further improvement of the PCE of our hybrid solar cells using a Al rear electrode, the formation of the BSF regions consisting of a heavily-doped $\mathrm{n}^{+}$layer at the Si back surface/rear electrode interface $[29,35]$ in order to prevent the ingress of holes toward trap levels at the interface regions, and the insertion of a hole-injection (and electron-blocking) layer at the PEDOT:PSS/front electrode interface [13] and/or an electron-injection (and hole-blocking) layer at the Si back surface/rear electrode interface [36] in order to reduce barrier height might be effective in enhancing the carrier transfer efficiency from the Si/PEDOT:PSS regions to each electrode. Introducing these approaches for our hybrid solar cells has the potential to acquire the cell performance comparable to the Si nanowire/PEDOT:PSS hybrid solar cells with high PCE of more than $11 \%$.

\section{Conclusions}

We demonstrated the effects of PEDOT:PSS coverage and the rear electrode on the photovoltaic performance of hybrid solar cells consisting of micro-pyramidal-structured Si/PEDOT:PSS composites. 
The adhesiveness of the PEDOT:PSS layer on the micro-pyramidal surfaces was dramatically improved by increasing the spin speed to $7500 \mathrm{rpm}$. The good adhesiveness of the PEDOT:PSS coverage had a significant effect on the antireflection capability related to the carrier generation, and the favorable separation of photogenerated charge carriers was realized. Using an $\mathrm{Al}$ rear electrode facilitated the efficient collection of separated electrons, improving both $J_{s c}$ and $V_{o c}$. The hybrid solar cells with optimal PEDOT:PSS layer and Al rear electrode achieved the best photovoltaic performance, with a PCE of $8.25 \%$. The present approach provides important information for improving the photovoltaic performance of $\mathrm{Si}$ /organic polymer hybrid solar cells with a micro-textured surface.

Acknowledgments: This work was supported in part by JSPS KAKENHI Grant Number 26390105 in Japan and Research Institute for Science and Technology of Tokyo Denki University Grant Number Q15E-01 in Japan.

Author Contributions: Keisuke Sato designed the experiments; Yuuki Sugano and Keisuke Sato performed the experiments and analyzed the results; Keisuke Sato, Naoki Fukata, and Kenji Hirakuri gave some useful suggestions for this work; Yuuki Sugano drafted the manuscript; Keisuke Sato finalized the manuscript.

Conflicts of Interest: The authors declare no conflict of interest.

\section{References}

1. Fthenakis, V.M.; Kim, H.C. Greenhouse-gas emissions from solar electric- and nuclear power: A life-cycle study. Energy Policy 2007, 35, 2549-2557. [CrossRef]

2. Jay, F.; Muñoz, D.; Desrues, T.; Pihan, E.; Amaral de Oliveira, V.; Enjalbert, N.; Jouini, A. Advanced process for n-type mono-like silicon a-Si:H/c-Si heterojunction solar cells with 21.5\% efficiency. Sol. Energy Mater. Sol. Cells 2014, 130, 690-695. [CrossRef]

3. Dwivedi, N.; Kumar, S.; Bisht, A.; Patel, K.; Sudhakar, S. Simulation approach for optimization of device structure and thickness of HIT solar cells to achieve 27\% efficiency. Sol. Energy 2013, 88, 31-41. [CrossRef]

4. Eser, E.; Ramaprasad, K.R.; Volltrauer, H.; Ramos, F.; Gau, S.C.; Vos, R. Long-term stability of amorphous silicon solar cells and modules. Sol. Cells 1987, 21, 25-39. [CrossRef]

5. Amine, A.; Yassaa, N.; Boudjema, R.; Aliouat, B. A new method for cost of renewable energy production in Algeria: Integrate all benefits drawn from fossil fuel savings. Renew. Sustain. Energy Rev. 2016, 56, 1150-1157.

6. Louwen, A.; Sark, W.V.; Schropp, R.; Faaij, A. A cost roadmap for silicon heterojunction solar cells. Sol. Energy Mater. Sol. Cells 2016, 147, 295-314. [CrossRef]

7. Brabec, C.J. Organic photovoltaics: Technology and market. Sol. Energy Mater. Sol. Cells 2004, 83, 273-292. [CrossRef]

8. Lee, T.-W.; Chung, Y. Control of the surface composition of a conducting-polymer complex film to tune the work function. Adv. Funct. Mater. 2008, 18, 2246-2252. [CrossRef]

9. Huang, J.; Miller, P.F.; Wilson, J.S.; Mello, A.J.D.; Mello, J.C.D.; Bradley, D.D.C. Investigation of the effects of doping and post-deposition treatments on the conductivity, morphology, and work function of poly(3,4-ethylenedioxythiophene)/poly(styrene sulfonate) films. Adv. Funct. Mater. 2005, 15, 290-296. [CrossRef]

10. He, L.; Jiang, C.; Wang, H.; Lai, D.; Rusli, E. High efficiency planar Si/organic heterojunction hybrid solar cells. Appl. Phys. Lett. 2012, 100, 073503. [CrossRef]

11. Liu, Q.; Imamura, T.; Hiate, T.; Khatri, I.; Tang, Z.; Ishikawa, R.; Ueno, K.; Shirai, H. Optical anisotropy in solvent-modified poly(3,4-ethylenedioxythiophene):poly(styrenesulfonic acid) and its effect on the photovoltaic performance of crystalline silicon/organic heterojunction solar cells. Appl. Phys. Lett. 2013, 102, 243902. [CrossRef]

12. Thomas, J.P.; Leung, K.T. Defect-minimized PEDOT:PSS/planar-Si solar cell with very high efficiency. Adv. Funct. Mater. 2014, 24, 4978-4985. [CrossRef]

13. Liu, R.; Lee, S.-T.; Sun, B. $13.8 \%$ efficiency hybrid $\mathrm{Si}$ /organic heterojunction solar cells with $\mathrm{MoO}_{3}$ film as antireflection and inversion induced layer. Adv. Mater. 2014, 26, 6007-6012. [CrossRef] [PubMed]

14. Xu, T.; Qiao, Q. Conjugated polymer-inorganic semiconductor hybrid solar cells. Energy Environ. Sci. 2011, 4, 2700-2720. [CrossRef]

15. Fan, X.; Zhang, M.; Wang, X.; Yang, F.; Meng, X. Recent progress in organic-inorganic hybrid solar cells. J. Mater. Chem. A 2013, 1, 8694-8709. [CrossRef] 
16. Chen, T.-G.; Huang, B.-Y.; Chen, E.-C.; Yu, P.; Meng, H.-F. Micro-textured conductive polymer/silicon heterojunction photovoltaic devices with high efficiency. Appl. Phys. Lett. 2012, 101, 033301. [CrossRef]

17. Arabloo, F.; Javadpour, S.; Memarzadeh, R.; Panahi, F.; Davazdah Emami, M.; Shariat, M.H. The interaction of carbon monoxide to Fe(III)(salen)-PEDOT:PSS composite as a gas sensor. Synth. Met. 2015, 209, $192-199$. [CrossRef]

18. Wakizaka, D.; Fushimi, T.; Ohkita, H.; Ito, S. Hole transport in conducting ultrathin films of PEDOT:PSS prepared by layer-by-layer deposition technique. Polymer 2004, 45, 8561-8565. [CrossRef]

19. Macfarlane, G.G.; Mclean, T.P.; Quarrington, J.E.; Roberts, V. Exciton and phonon effects in the absorption spectra of germanium and silicon. J. Phys. Chem. Solids 1959, 8, 388-392. [CrossRef]

20. Read, A.J.; Needs, R.J.; Nash, K.J.; Canham, L.T.; Calcott, P.D.J.; Qteish, A. First-principles calculations of the electronic properties of silicon quantum wires. Phys. Rev. Lett. 1993, 70, 2050. [CrossRef]

21. Green, M.A.; Zhao, J.; Wang, A.; Reece, P.J.; Gal, M. Efficient silicon light-emitting diodes. Nature 2001, 412, 805-808. [CrossRef] [PubMed]

22. Yang, L.; Liu, Y.; Chen, W.; Wang, Y.; Liang, H.; Mei, A.; Kuznetsov, A.; Du, X. Interface Engineering of high efficiency organic-silicon heterojunction solar cells. ACS Appl. Mater. Interfaces 2016, 8, 26-30. [CrossRef] [PubMed]

23. Liu, C.-Y.; Holman, Z.C.; Kortshagen, U.R. Optimization of Si NC/P3HT hybrid solar cells. Adv. Funct. Mater. 2010, 20, 2157-2164. [CrossRef]

24. Ozdemir, B.; Kulakci, M.; Turan, R.; Unalan, H.E. Silicon nanowire-poly(3,4-ethylenedioxythiophene)poly(styrenesulfonate) heterojunction solar cells. Appl. Phys. Lett. 2011, 99, 113510. [CrossRef]

25. He, L.; Jiang, C.; Rusli, E.; Lai, D.; Wang, H. Highly efficient Si-nanorods/organic hybrid core-sheath heterojunction solar cells. Appl. Phys. Lett. 2011, 99, 021104. [CrossRef]

26. Leem, D.-S.; Edwards, A.; Faist, M.; Nelson, J.; Bradley, D.D.C.; Mello, J.C. Efficient organic solar cells with solution-processed silver nanowire electrodes. Adv. Mater. 2011, 23, 4371-4375. [CrossRef] [PubMed]

27. Lim, D.C.; Kim, K.-D.; Park, S.-Y.; Hong, E.M.; Seo, H.O.; Lim, J.H.; Lee, K.H.; Jeong, Y.; Song, C.; Lee, E.; et al. Towards fabrication of high-performing organic photovoltaics: New donor-polymer, atomic layer deposited thin buffer layer and plasmonic effects. Energy Environ. Sci. 2012, 5, 9803-9807. [CrossRef]

28. Kuo, C.Y.; Gau, C. Arrangement of band structure for organic-inorganic photovoltaics embedded with silicon nanowire arrays grown on indium tin oxide glass. Appl. Phys. Lett. 2009, 95, 053302. [CrossRef]

29. Thiyagu, S.; Hsueh, C.-C.; Liu, C.-T.; Syu, H.-J.; Lin, T.-C.; Lin, C.-F. Hybrid organic-inorganic heterojunction solar cells with $12 \%$ efficiency by utilizing flexible film-silicon with a hierarchical surface. Nanoscale 2014, 6 , 3361-3366. [CrossRef] [PubMed]

30. Sato, K.; Dutta, M.; Fukata, N. Inorganic/organic hybrid solar cells: Optimal carrier transport in vertically aligned slicon nanowire arrays. Nanoscale 2014, 6, 6092-6101. [CrossRef] [PubMed]

31. Dean, J.A. Section 4 properties of atoms, radicals, and bonds. In Lange's Handbook of Chemistry, 15th ed.; McGraw-Hill, Inc.: New York, NY, USA, 1999.

32. Zhang, F.; Sun, B.; Song, T.; Zhu, X.; Lee, S. Air stable, efficient hybrid photovoltaic devices based on poly(3-hexylthiophene) and silicon nanostructures. Chem. Mater. 2011, 23, 2084-2090. [CrossRef]

33. Lei, X.; Zhang, F.; Song, T.; Sun, B. P-type doping effect on the performance of organic-inorganic hybrid solar cells. Appl. Phys. Lett. 2011, 99, 233305. [CrossRef]

34. Liu, Q.; Khatri, I.; Ishikawa, R.; Ueno, K.; Shirai, H. Effects of molybdenum oxide molecular doping on the chemical structure of poly(3,4-ethylenedioxythiophene):poly(stylenesulfonate) and on carrier collection efficiency of silicon/poly(3,4-ethylenedioxythiphene):poly(stylenesulfonate) heterojunction solar cells. Appl. Phys. Lett. 2013, 102, 183503.

35. Jeong, S.; Garnett, E.C.; Wang, S.; Yu, Z.; Fan, S.; Brongersma, M.L.; McGehee, M.D.; Cui, Y. Hybrid silicon nanocone-polymer solar cells. Nano Lett. 2012, 12, 2971-2976. [CrossRef] [PubMed]

36. Zhang, Y.; Cui, W.; Zhu, Y.; Zu, F.; Liao, L.; Lee, S.-T.; Sun, B. High efficiency hybrid PEDOT:PSS/ nanostructured silicon schottky junction solar cells by doping-free rear contact. Energy Environ. Sci. 2015, 8, 297-302. [CrossRef]

(C) 2017 by the authors. Licensee MDPI, Basel, Switzerland. This article is an open access article distributed under the terms and conditions of the Creative Commons Attribution (CC BY) license (http://creativecommons.org/licenses/by/4.0/). 